\title{
Analysis of condenser shell side pressure drop based on the mechanical energy loss
}

\author{
ZENG Hui, MENG JiAn \& LI ZhiXin* \\ Key Laboratory of Thermal Science and Power Engineering of Ministry of Education, School of Aerospace, Tsinghua University, \\ Beijing 100084, China
}

Received May 16, 2012; accepted July 13, 2012

\begin{abstract}
The condenser performance is strongly affected by the tube arrangement. The steam pressure drop in the tube bundle influences the condenser back pressure, which is an important indicator of the condenser performance used to compare different condenser tube arrangements. The condenser shell side pressure drop is studied here using the mechanical energy loss of the steam flow in the condensers. The mechanical energy loss is due to the flow resistance of the tube bundle and the steam condensation. Three typical tube arrangements are analyzed numerically. The results show that a higher condenser shell side pressure drop for different tube arrangements always corresponds to a larger mechanical energy loss. The mechanical energy loss is mainly in the periphery of the tube bundle, indicating that the flow pattern and the mechanical energy losses are markedly determined by the tube bundle profile. The condenser shell side pressure drop can be reduced by reducing the total mechanical energy loss when the steam enters the tube bundle more uniformly. Thus, a well designed tube arrangement will reduce the mechanical energy loss, and also the shell side pressure drop.
\end{abstract}

power plant condenser, tube arrangement, shell side pressure drop, mechanical energy loss

Citation: Zeng H, Meng J A, Li Z X. Analysis of condenser shell side pressure drop based on the mechanical energy loss. Chin Sci Bull, 2012, 57: 4718-4725, doi: $10.1007 / \mathrm{s} 11434-012-5558-4$

Condensers, normally complex shell-and-tube heat exchangers, are the most important auxiliary equipments in a power plant. The thermal efficiency of power unit strongly depends on the condenser back pressure. A condenser has a large number of cooling tubes. For example, a condenser for a $300 \mathrm{MW}$ unit has approximately 20000 cooling tubes. The condenser performance is then highly dependent on the tube arrangement. The shell side pressure drop in the tube bundle is an important indicator of the condenser performance, which can be used to compare different condenser tube arrangements.

Power plant condenser designs are traditionally based on recommendations from previous designs and experimental results, often based on the standards developed by the Heat Exchange Institute (HEI Standards) [1]. However, this method does not take a number of factors into account, in-

\footnotetext{
*Corresponding author (email: lizhx@tsinghua.edu.cn)
}

cluding the tube arrangement. Numerical simulations of the fluid flow and heat transfer in condensers have been conducted by many researchers [2-10], with predictions agreeing well with experimental data. The advantage of numerical simulations is that they can give more detailed information on the fluid flow and heat transfer in the condenser, which provides a more effective means for condenser design. However, there is still a lack of a good optimization method for the tube arrangement, which can improve the condenser performance by reducing the shell side pressure drop.

Guo et al. [11] recently introduced a new physical quantity, entransy, for optimizing heat transfer processes, which describes the heat transfer ability of a body. The heat conduction equation is multiplied by the temperature to get an entransy balance equation, with $\lambda(\nabla T)^{2}$ as the entransy dissipation rate. The entransy dissipation rate is a measure of the irreversibility of a heat transfer process. Then, Guo et al. [11] proposed the extremum entransy dissipation principle 
for optimizing heat transfer processes where the extremum entransy dissipation corresponds to the optimal heat transfer performance when a thermodynamic cycle is not involved. This new principle has been applied to optimizing heat conduction [11-19], convective heat transfer [20-23], thermal radiation [24] processes and the heat transfer in heat exchanger [25-33], with all the studies confirming that the optimal results can be obtained based on the extremum entransy dissipation principle.

Chen et al. [34-36] analyzed the heat, mass and momentum transfer to extend the entransy concept to convective mass transfer and fluid flow processes. The mass entransy and momentum entransy dissipations were defined for optimizing convective mass transfer and fluid flow processes. The momentum entransy dissipation is equivalent to the mechanical energy loss during a fluid flow process.

In order to improve the condenser performance, this study analyzes the condenser shell side pressure drop based on the mechanical energy loss for the steam flow. Three typical tube arrangements are taken into consideration to study the impact of the tube arrangement on the condenser shell side pressure drop.

\section{Physical model}

Figure 1 shows the entire cross section of a condenser with the cap-shaped tube arrangement. The shell side of the condenser can be divided into two distinct flow regions, one is the tube bundle region where the cooling tubes are densely positioned, and the other is the free flow region. The steam exhaust from the turbine enters the condenser from the top and flows through the tube bundle to condense. The noncondensable gases (mainly air) and the non-condensed steam are extracted by the vents in the centre of each tube bundle. Baffles guide the steam flow. Some tube arrangements have an independent tube bundle in front of the vent, which is called air cooling region.

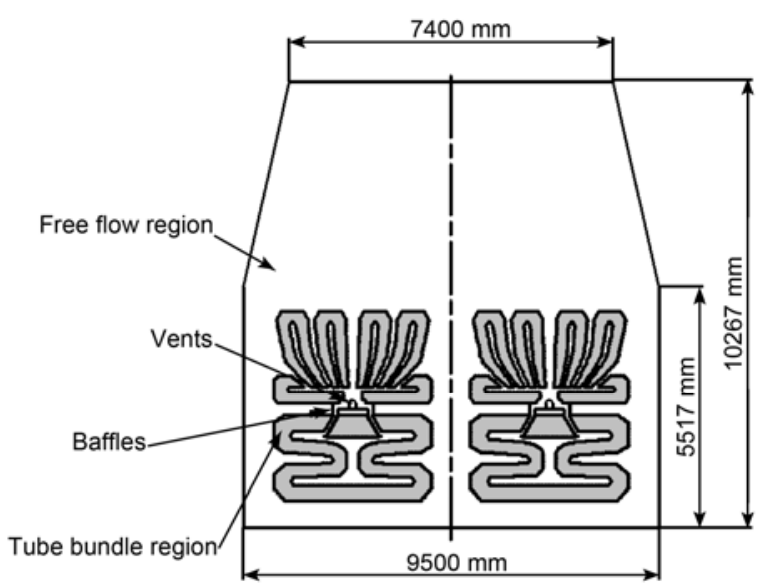

Figure 1 Condenser cross-section.
As in previous studies, the shell side of the condenser is modeled as a porous medium. An isotropic porosity, $\beta$, is defined as the ratio of the volume occupied by the fluid to the total volume. In the present work, $\beta=\beta_{\mathrm{f}}=1$ in the free flow region, and in the tube bundle region, where the tubes are laid out in an equilateral triangular pattern, $\beta=\beta_{\mathrm{t}}$, it is set to

$$
\beta_{\mathrm{t}}=1-\frac{\pi}{2 \sqrt{3}}\left(\frac{D_{\mathrm{o}}}{P_{\mathrm{t}}}\right)^{2},
$$

where $D_{\mathrm{o}}$ is the tube outer diameter and $P_{\mathrm{t}}$ is the tube pitch.

The complex shell side flow in the condenser is simplified as a two dimensional gas (a mixture of steam and air) flow through a porous medium. The two-dimensional steadystate governing equations for mass, momentum, and the air mass fraction in the Cartesian coordinate system are as follows [6].

Mass conservation equation for the steam and air mixture:

$$
\frac{\partial}{\partial x}(\beta \rho u)+\frac{\partial}{\partial y}(\beta \rho v)=-\beta \dot{m}
$$

Momentum conservation equations for the steam and air mixture:

$$
\begin{aligned}
& \frac{\partial}{\partial x}(\beta \rho u u)+\frac{\partial}{\partial y}(\beta \rho v u)= \\
& \frac{\partial}{\partial x}\left[\beta\left(\mu_{\mathrm{t}}+\mu\right) \frac{\partial u}{\partial x}\right]+\frac{\partial}{\partial y}\left[\beta\left(\mu_{\mathrm{t}}+\mu\right) \frac{\partial u}{\partial y}\right]-\beta \frac{\partial P}{\partial x}-\beta \dot{m} u-\beta F_{u},
\end{aligned}
$$

$$
\begin{aligned}
& \frac{\partial}{\partial x}(\beta \rho u v)+\frac{\partial}{\partial y}(\beta \rho v v)= \\
& \frac{\partial}{\partial x}\left[\beta\left(\mu_{\mathrm{t}}+\mu\right) \frac{\partial v}{\partial x}\right]+\frac{\partial}{\partial y}\left[\beta\left(\mu_{\mathrm{t}}+\mu\right) \frac{\partial v}{\partial y}\right]-\beta \frac{\partial P}{\partial y}-\beta \dot{m} v-\beta F_{v} ;
\end{aligned}
$$

Conservation of air mass fraction:

$$
\begin{aligned}
& \frac{\partial}{\partial x}\left(\beta \rho u Y_{\mathrm{a}}\right)+\frac{\partial}{\partial y}\left(\beta \rho v Y_{\mathrm{a}}\right)= \\
& \frac{\partial}{\partial x}\left[\beta \rho\left(D_{\mathrm{t}}+D\right) \frac{\partial Y_{\mathrm{a}}}{\partial x}\right]+\frac{\partial}{\partial y}\left[\beta \rho\left(D_{\mathrm{t}}+D\right) \frac{\partial Y_{\mathrm{a}}}{\partial y}\right],
\end{aligned}
$$

where $\dot{m}$ is the steam condensate rate per unit volume, $F_{u}$ and $F_{v}$ are the flow resistance force components in the $x$ and $y$ directions due to the tube bundle, $\mu$ is the dynamic viscosity, $D$ is the diffusivity of air in the vapor, $\mu_{\mathrm{t}}$ is the turbulent dynamic viscosity, $D_{\mathrm{t}}$ is the turbulent diffusivity of air in the vapor, and $Y_{\mathrm{a}}$ is the air mass fraction.

\section{Mechanical energy loss for the condenser shell side flow}

The expression of the mechanical energy loss can be derived 
from the governing equations. The porosities in both the tube bundle region and the free flow region are uniform and change only at the interface between the two regions, so the mechanical energy dissipation caused by the porosity variation is neglected. Multiplying eqs. (3) and (4) by the velocity components $u$ and $v$ respectively gives

$$
\begin{aligned}
& u \frac{\partial}{\partial x}(\beta \rho u u)+u \frac{\partial}{\partial y}(\beta \rho v u)= \\
& u \frac{\partial}{\partial x}\left[\beta\left(\mu_{\mathrm{t}}+\mu\right) \frac{\partial u}{\partial x}\right]+u \frac{\partial}{\partial y}\left[\beta\left(\mu_{\mathrm{t}}+\mu\right) \frac{\partial u}{\partial y}\right]-\beta u \frac{\partial P}{\partial x} \\
& \quad-\beta \dot{m} u^{2}-\beta F_{u} u, \\
& v \frac{\partial}{\partial x}(\beta \rho u v)+v \frac{\partial}{\partial y}(\beta \rho v v)= \\
& v \frac{\partial}{\partial x}\left[\beta\left(\mu_{\mathrm{t}}+\mu\right) \frac{\partial v}{\partial x}\right]+v \frac{\partial}{\partial y}\left[\beta\left(\mu_{\mathrm{t}}+\mu\right) \frac{\partial v}{\partial y}\right]-\beta v \frac{\partial P}{\partial y} \\
& \quad-\beta \dot{m} v^{2}-\beta F_{v} v .
\end{aligned}
$$

Combining eqs. (6) and (7) and make some transformations, and substituting eq. (2) into it yields

$$
\begin{aligned}
& \frac{\partial}{\partial x}\left[\beta \rho u \frac{1}{2}\left(u^{2}+v^{2}\right)\right]+\frac{\partial}{\partial y}\left[\beta \rho v \frac{1}{2}\left(u^{2}+v^{2}\right)\right]+\frac{\partial(\beta P u)}{\partial x} \\
& +\frac{\partial(\beta P v)}{\partial y} \\
& =-\beta \frac{P}{\rho} \dot{m}-\frac{1}{2} \beta\left(u^{2}+v^{2}\right) \dot{m}-\beta\left(F_{u} u+F_{v} v\right)-\varphi_{\mu},
\end{aligned}
$$

where $\varphi_{\mu}$ is the viscous dissipation function, which is expressed as

$$
\varphi_{\mu}=\left(\mu_{\mathrm{t}}+\mu\right)\left[2\left(\frac{\partial u}{\partial x}\right)^{2}+2\left[\frac{\partial v}{\partial y}\right]^{2}+\left(\frac{\partial u}{\partial y}+\frac{\partial v}{\partial x}\right)^{2}\right]
$$

Integrating eq. (8) over the whole flow region and using Gauss' rule gives

$$
\begin{aligned}
& \int_{A}\left[\frac{1}{2} \rho\left(u^{2}+v^{2}\right)(u \vec{i}+v \vec{j}) \cdot \vec{n}\right] \mathrm{d} A+\iint_{A}[P(u \vec{i}+v \vec{j}) \cdot \vec{n}] \mathrm{d} A \\
& =-\iiint_{V}\left[\beta \frac{P}{\rho} \dot{m}+\frac{1}{2} \beta\left(u^{2}+v^{2}\right) \dot{m}+\beta\left(F_{u} u+F_{v} v\right)+\varphi_{\mu}\right] \mathrm{d} V .
\end{aligned}
$$

Eq. (10) is the mechanical energy conservation equation for the condenser shell side flow. The left side of eq. (10) is the mechanical energy transport through the boundary, and the right side is the mechanical energy loss during the fluid flow.

Since most of the steam will condense in the condenser, the mechanical energy transport through the vents can be neglected. Thus, the mechanical energy conservation equation can be simplified to

$$
\begin{aligned}
& \left(\frac{1}{2} U_{\mathrm{i}}^{2}+\frac{P_{i}}{\rho}\right) Q_{\mathrm{i}} \\
& =\iiint_{V}\left[\beta \frac{P}{\rho} \dot{m}+\frac{1}{2} \beta\left(u^{2}+v^{2}\right) \dot{m}+\beta\left(F_{u} u+F_{v} v\right)+\varphi_{\mu}\right] \mathrm{d} V,
\end{aligned}
$$

where $U_{\mathrm{i}}$ and $Q_{\mathrm{i}}$ are the inlet velocity and mass flow rate determined by the condenser operating conditions. $P_{\mathrm{i}}$ is the inlet pressure.

The absolute pressure in eq. (11) can be changed to a relative pressure with the vent pressure, $P_{\mathrm{o}}$, as the reference:

$$
\bar{P}=P-P_{\text {o }} \text {. }
$$

The relative inlet pressure, $\bar{P}_{\mathrm{i}}$, is then equal to the shell side pressure drop, $\Delta P$. Then eq. (11) can be written as

$$
\begin{aligned}
& \left(\frac{1}{2} U_{\mathrm{i}}^{2}+\frac{\Delta P}{\rho}\right) Q_{\mathrm{i}} \\
& =\iiint_{V}\left[\beta \frac{\bar{P}}{\rho} \dot{m}+\frac{1}{2} \beta\left(u^{2}+v^{2}\right) \dot{m}+\beta\left(F_{u} u+F_{v} v\right)+\varphi_{\mu}\right] \mathrm{d} V .
\end{aligned}
$$

The physical meanings of each term in the mechanical energy conservation equation, eq. (13), are

$\frac{1}{2} U_{\mathrm{i}}^{2} Q_{\mathrm{i}}$, the kinetic energy input through the inlet;

$\frac{\Delta P}{\rho} Q_{\mathrm{i}}$, the potential energy input through the inlet;

$\iiint_{V}\left(\beta \frac{\bar{P}}{\rho} \dot{m}\right) \mathrm{d} V$, the potential energy loss due to the steam condensation in the tube bundle region;

$\iiint_{V}\left[\frac{1}{2} \beta\left(u^{2}+v^{2}\right) \dot{m}\right] \mathrm{d} V$, the kinetic energy loss due to the steam condensation in the tube bundle region;

$\iiint_{V}\left[\beta\left(F_{u} u+F_{v} v\right)\right] \mathrm{d} V$, the mechanical energy loss due to the flow resistance in the tube bundle region;

$\iiint_{V} \varphi_{\mu} \mathrm{d} V$, the mechanical energy loss due to viscous dissipation in the free flow region.

Because there is steam condensation during the fluid flow process, the total mechanical energy loss consists of not only the mechanical energy loss due to the flow resistance, but also the mechanical energy loss due to the steam condensation.

Based on eq. (13), the total mechanical energy input, $E_{\mathrm{i}}$, and the total mechanical energy loss rate, $\Phi_{\mathrm{e}}$, can be defined as

$$
E_{\mathrm{i}}=\left(\frac{1}{2} U_{\mathrm{i}}^{2}+\frac{\Delta P}{\rho}\right) Q
$$




$$
\Phi_{\mathrm{e}}=\iiint_{V} \varphi_{\mathrm{e}} \mathrm{d} V
$$

where

$$
\varphi_{\mathrm{e}}=\beta \frac{\bar{P}}{\rho} \dot{m}+\frac{1}{2} \beta\left(u^{2}+v^{2}\right) \dot{m}+\beta\left(F_{u} u+F_{v} v\right)+\varphi_{\mu}
$$

is the mechanical energy loss rate.

In eq. (13), $U_{\mathrm{i}}$ and $Q_{\mathrm{i}}$ are fixed values for the given operating conditions, indicating that the condenser shell side pressure drop can be measured by the mechanical energy loss. The mechanical energy loss is related to the tube arrangement, so it provides a way to study the impact of the tube arrangement on the condenser shell side pressure drop.

\section{Numerical analyses of typical tube arrange- ments}

Three typical tube arrangements are considered to study the impact of the tube arrangement on the condenser shell side pressure drop. Figure 2 shows the tube arrangements where type A is a cap-shaped tube arrangement, type B is a doublepeak-shaped tube arrangement, and type $\mathrm{C}$ is a lozengeshaped tube arrangement. Since all condensers are symmetric across their center planes, Figure 2 only shows half of each tube bundle. Each condenser has double tube-passes,

so each tube bundle is formed by two parts with equal numbers of tubes. The cooling water enters into the lower tube bundle and then exits from the upper tube bundle.

The condenser shell side flow is complex, so the flow parameters distribution has no analytical solution. Thus, numerical method is used to analyze the three typical tube arrangements based on the mechanical energy loss. The condenser geometries and operating parameters are listed in Table 1 . The geometry and boundary conditions are symmetric, so only the left half of the condenser was simulated.

The steam condensate rate per unit volume, $\dot{m}$, and the flow resistance forces, $F_{u}$ and $F_{v}$, in eqs. (2)-(5) were taken from Zhang and Bokil [6]. The RNG $k-\varepsilon$ model [37] was used to model the turbulence through the turbulent viscosity, $\mu_{\mathrm{t}}$, and the turbulent diffusivity of air, $D_{\mathrm{t}}$, in the vapor.

The boundary conditions for the computational domain are

Steam inlet: The velocity and air mass fraction are given at the inlet boundary;

Vent: The pressures at all the vents are all the same;

Walls: The shell walls and baffles of the condenser were assumed to be non-slip and impermeable;

Symmetry plane: Along the center symmetry line the derivatives with respect to the cross-stream direction of all field variables were set to zero except for the cross flow velocity which is set to zero.

The simulations were performed using the commercial

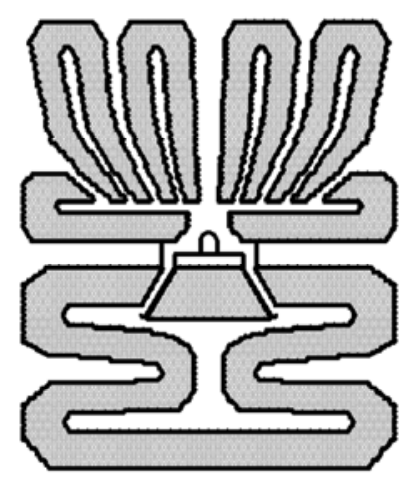

(a)

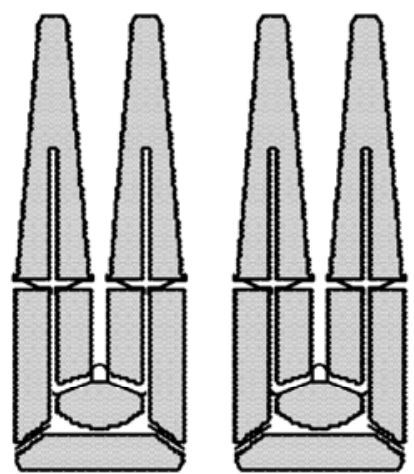

(b)

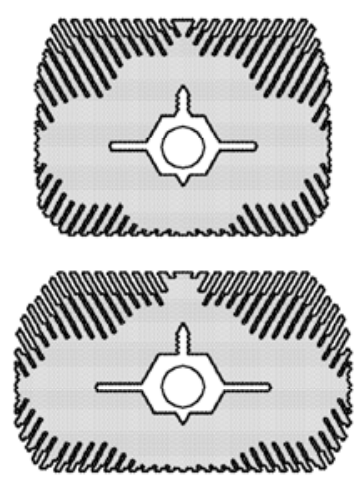

(c)

Figure 2 Three typical tube arrangements. (a) Cap-shaped tube arrangement; (b) double-peak-shaped tube arrangement; (c) Lozenge-shaped tube arrangement.

Table 1 Condenser geometric and operating parameters

\begin{tabular}{ll||l}
\hline \multicolumn{2}{c||}{ Geometric parameters } & \multicolumn{1}{c}{ Operating parameters } \\
\hline Tube material & TP $304 \mathrm{~L}$ & Inlet steam mass rate \\
Tube number & 22374 & Inlet steam dryness fraction \\
Tube length & $10.84 \mathrm{~m}$ & Air in-leakage mass fraction \\
Outer tube diameter & $25 \mathrm{~mm}$ & Venting pressure \\
Inner tube diameter & $24 \mathrm{~mm}$ & Cooling water flow rate \\
Tube pitch & $32 \mathrm{~mm}$ & Inlet cooling water temperature \\
& & Tube cleanliness factor \\
\hline
\end{tabular}


software FLUENT 6.2. The discretization used the SIMPLEC algorithm with the QUICK scheme.

Non-uniform quadrilateral grids were generated using Gambit 2.0, with fine elements in the tube bundle region and coarse elements in the external free flow region. As an example, the grids near the boundary between the tube bundle region and the free flow region of condenser for tube arrangement A are shown in Figure 3. Calculation results with different element numbers of condenser for tube arrangement $\mathrm{A}$ are listed in Table 2 to test the grid independence. The results showed that the element number of about 900000 is sufficiently fine to result in a grid-independent solution.

\subsection{Mechanical energy loss for tube arrangement A}

Tube arrangement $\mathrm{A}$ is a cap-shaped tube arrangement. The tubes are arranged as a continuously closed band, and which is curved to form steam inlet channels. According to the simulation result, the total mechanical energy input, $E_{\mathrm{i}}$, is $852 \mathrm{~kW}$ and the total mechanical energy loss, $\Phi_{\mathrm{e}}$, is $746 \mathrm{~kW}$. The total mechanical energy loss is only $10 \%$ less than the total mechanical energy input, showing that $\Phi_{\mathrm{e}}$ can be used to approximately measure the shell side pressure drop. The difference between $\Phi_{\mathrm{e}}$ and $E_{\mathrm{i}}$ is the mechanical energy loss due to the change of porosity when the steam enters the tube bundle region from the free flow region.

Figure 4 shows the flow streamlines in the condenser for tube arrangement $\mathrm{A}$. The steam enters the condenser at the

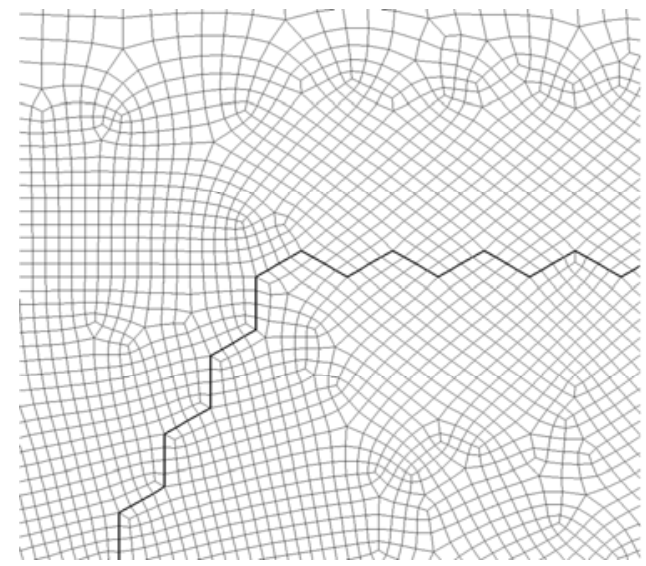

Figure 3 Grids near the boundary between the tube bundle region and the free flow region.

Table 2 Grid independence test results

\begin{tabular}{cc}
\hline Element number & $\Delta P(\mathrm{~Pa})$ \\
\hline 374684 & 293.22 \\
618061 & 294.32 \\
952343 & 297.78 \\
1498328 & 298.51 \\
\hline
\end{tabular}

top with a uniform downward velocity, flows around the tube bundle and enters into the tube bundle from all around the periphery. Because of the steam condensation in the tube bundle, the steam velocity decreases rapidly as the steam flows through tube bundle towards the vent. The side wall causes the flow resistance in the left channel to be larger than that in the right channel where the symmetry plane has no effect on the flow resistance. The steam flowing through the right main channel turns into the bottom channel and then flows upward into the left main channel until reaching the middle part of the lower tube bundle. The steam enters the tube bundle intensively in the top part of the upper tube bundle, in the bottom part of the lower tube bundle and in the left part of the lower tube bundle. Some vortices develop near the right channel where the pressure is lower. Not much of the steam flow through the right channel enters the tube bundle, indicating that this part of tube bundle is not effectively used.

The mechanical energy losses in the condenser for tube arrangement $\mathrm{A}$ is shown in Figure 5. The viscous dissipation in the free flow region is not so high where the maximum viscous dissipation in the free flow region is about $1000 \mathrm{~W} / \mathrm{m}^{3}$, and the viscous dissipation in most of the free flow region is lower than $50 \mathrm{~W} / \mathrm{m}^{3}$. As the steam enters the tube bundle to condense with a rapidly decreasing velocity and pressure, the mechanical energy loss decreases from $20000 \mathrm{~W} / \mathrm{m}^{3}$ to less than $50 \mathrm{~W} / \mathrm{m}^{3}$ near the vent. The region with mechanical energy losses higher than $20000 \mathrm{~W} / \mathrm{m}^{3}$ is where the steam enters into the tube bundle. Besides the region near the vents, along the right side of the tube bundle in the right channel, there are also some regions with mechanical energy losses lower than $50 \mathrm{~W} / \mathrm{m}^{3}$.

The pressure distribution of condenser for tube arrangement A is shown in Figure 6. The pressure plotted in the figure is the relative pressure with the vent pressure as reference. Figure 6 shows that the pressure drops rapidly in the

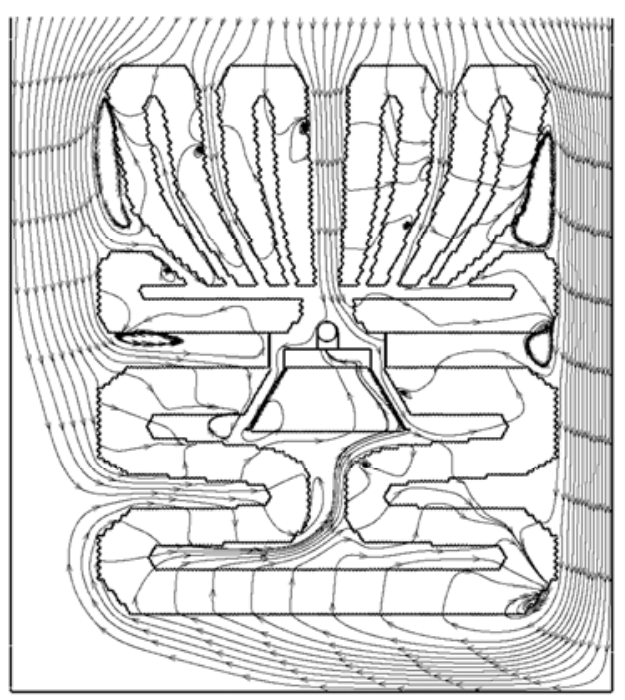

Figure 4 Streamlines of condenser for tube arrangement A. 


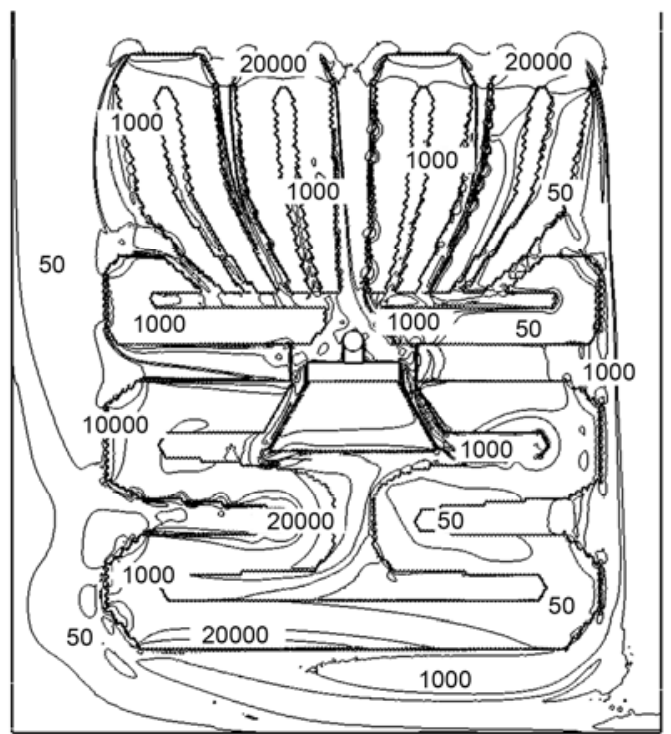

Figure 5 Mechanical energy losses of condenser for tube arrangement A $\left(\mathrm{W} / \mathrm{m}^{3}\right)$.

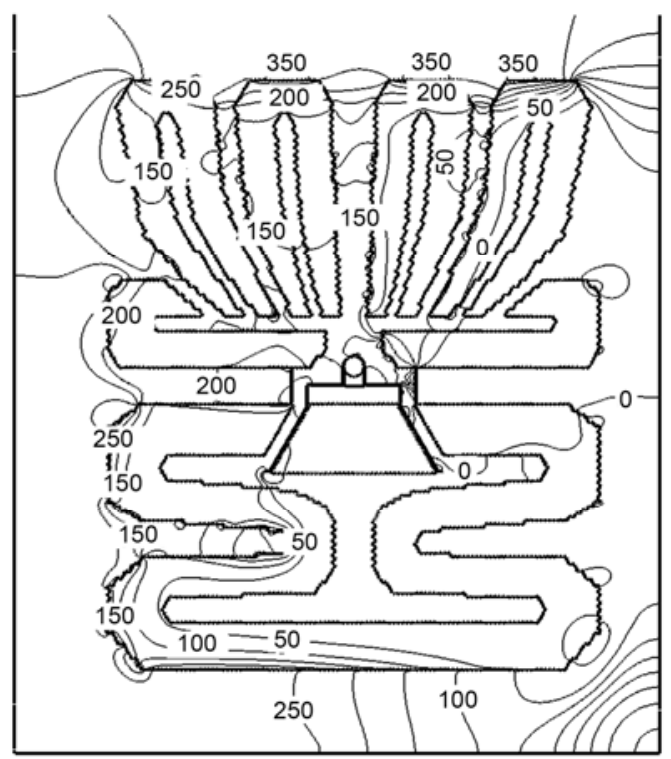

Figure 6 Pressure distribution of condenser for tube arrangement A.

regions with high mechanical energy losses. That means high mechanical energy loss corresponds to large pressure drop in the condenser.

The mechanical energy loss distribution in the condenser for tube arrangement $\mathrm{A}$ is not uniform. The total mechanical energy loss is quite large so the condenser shell side pressure drop is quite high. The shell side pressure drop of the condenser for tube arrangement A is $298 \mathrm{~Pa}$.

\subsection{Mechanical energy loss for tube arrangement B}

Tube arrangement B is a double-peak-shaped tube arrangement with each half of the condenser having two identical tube bundles, each bundle is shaped as two connected peaks. The steam channels are taper-shaped. Figure 7 shows the flow streamlines of the condenser for tube arrangement B. The steam first flows downward through the middle and right channels, then enters into the bottom main channel and finally flows upward through the left main channel to the top-left corner of the tube bundle. The steam flow through the tapered channels uniformly enters the tube bundle at different positions all along the bundle. There are some small vortices in the lower tube bundle and in the middle and right channels.

The mechanical energy losses in the condenser for tube arrangement B are shown in Figure 8. The double-peakshaped tube arrangement reduces the maximum mechanical energy loss compared to tube arrangement A. There are only small regions with mechanical energy losses higher than $10000 \mathrm{~W} / \mathrm{m}^{3}$ and the mechanical energy losses in most of the tube bundle are between $1000-5000 \mathrm{~W} / \mathrm{m}^{3}$. There are some small regions with mechanical energy losses lower than $50 \mathrm{~W} / \mathrm{m}^{3}$ in the periphery of the lower tube bundle, showing that the mechanical energy loss distribution there is not reasonable.

The distribution of the mechanical energy loss of the condenser for tube arrangement B is much more uniform than for tube arrangement $\mathrm{A}$. The maximum mechanical energy loss is lower and the regions in the periphery of the tube bundle with low mechanical energy losses are smaller. Thus, the total mechanical energy loss is $370 \mathrm{~kW}$ and the shell side pressure is $130 \mathrm{~Pa}$ of the condenser for tube arrangement $\mathrm{B}$, which are both lower than of the condenser for tube arrangement $\mathrm{A}$.

\subsection{Mechanical energy losses for tube arrangement $\mathrm{C}$}

Tube arrangement $\mathrm{C}$ is a lozenge-shaped tube arrangement

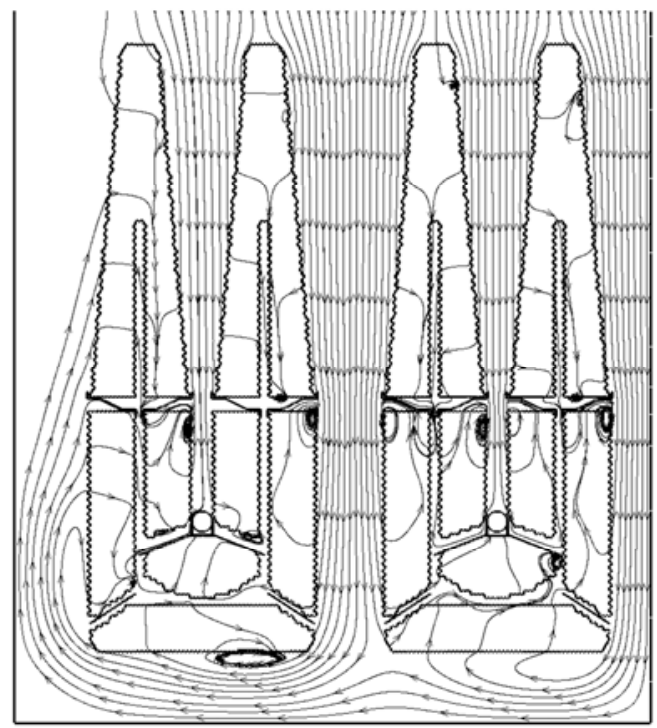

Figure 7 Streamlines of condenser for tube arrangement B. 


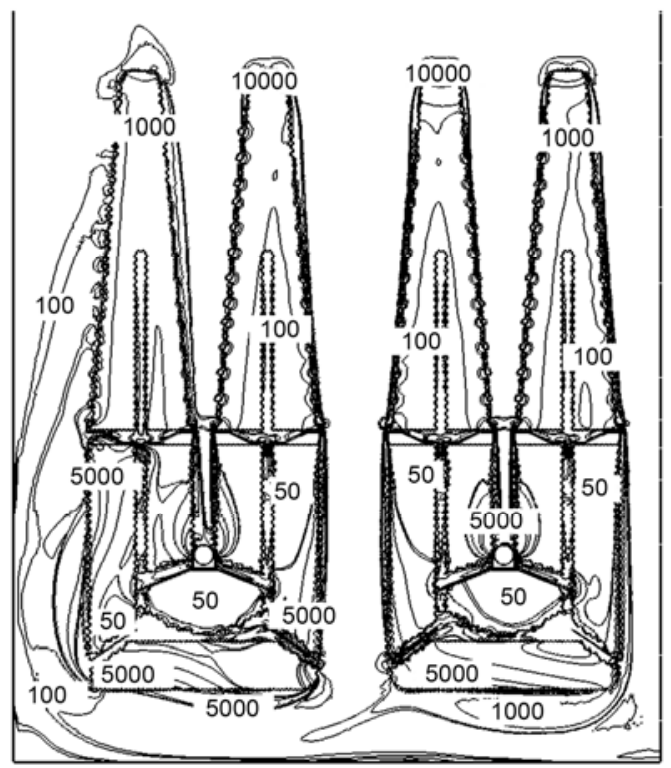

Figure 8 Mechanical energy losses of condenser for tube arrangement B $\left(\mathrm{W} / \mathrm{m}^{3}\right)$.

with each half of the condenser having two tube bundles, which are similar but not the same. The tube bundle is approximately lozenge-shaped with tubes removed in the tube bundle periphery to form tiny secondary branch channels. Figure 9 shows the flow streamlines in the condenser for tube arrangement $\mathrm{C}$. The steam flows downward in the right main channel, but in the left main channel the steam only flows half way and then turns into the horizontal main channel, and then flows to the right main channel. The two steam flows mingle and flow around the lower tube bundle until reaching the left-top corner. There are two large vortices with one in the lower-left part of the upper tube bundle

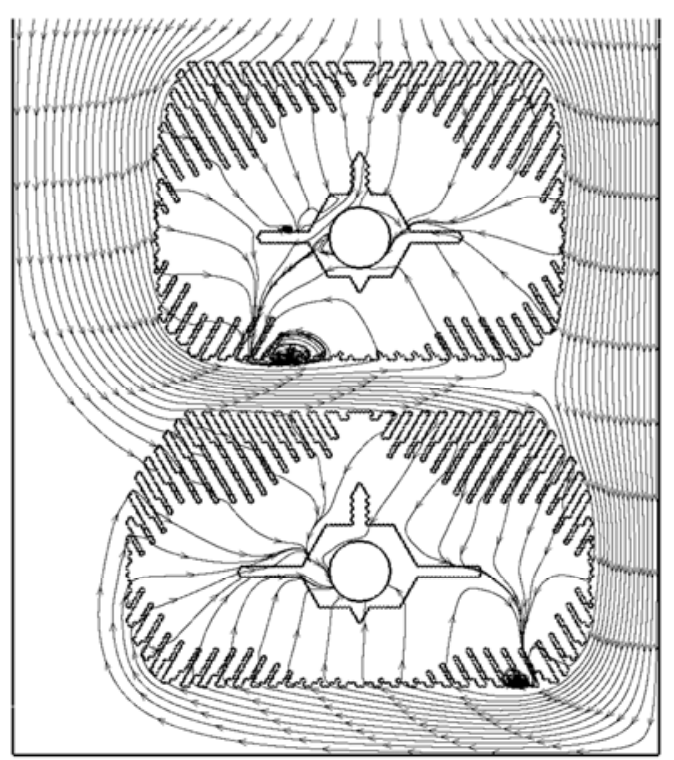

Figure 9 Streamlines of condenser for tube arrangement C. and the other in the lower-right part of the lower tube bundle, forming a large area of the tube bundle region where the steam does not flow to the vent and the new steam cannot enter.

The mechanical energy losses in the condenser for tube arrangement $\mathrm{C}$ are shown in Figure 10. The regions in the center of each bundle are connected with the vortices to form large areas with mechanical energy loss lower than $50 \mathrm{~W} / \mathrm{m}^{3}$. The region with mechanical energy losses higher than $20000 \mathrm{~W} / \mathrm{m}^{3}$ is larger than in the condensers for tube arrangements $\mathrm{A}$ and $\mathrm{B}$.

The mechanical energy loss distribution in the condenser for tube arrangement $\mathrm{C}$ is reasonable in part of tube bundle region, but the regions with low mechanical energy losses are too large, as well as the regions are with high mechanical energy losses. The total mechanical energy loss is 801 $\mathrm{kW}$ and the shell side pressure is $314 \mathrm{~Pa}$ of the condenser for tube arrangement $\mathrm{C}$, which is higher than for tube arrangement $\mathrm{A}$.

Among the three tube arrangements, condenser for tube arrangement $\mathrm{B}$ has the most uniform distribution of the mechanical energy loss, i.e. the regions with high and low mechanical energy losses are both small, so the total mechanical energy loss and the shell side pressure drop are both lower than for the other two tube arrangements. The mechanical energy loss distributions for tube arrangements $\mathrm{A}$ and $\mathrm{C}$ are not so reasonable with regions of low mechanical energy losses in the main condensation tube bundle, so the regions with high mechanical energy losses are very large. The mechanical energy losses are concentrated in the periphery of the tube bundle, indicating that the flow pattern and the mechanical energy losses are markedly determined by the profile of the tube bundle.

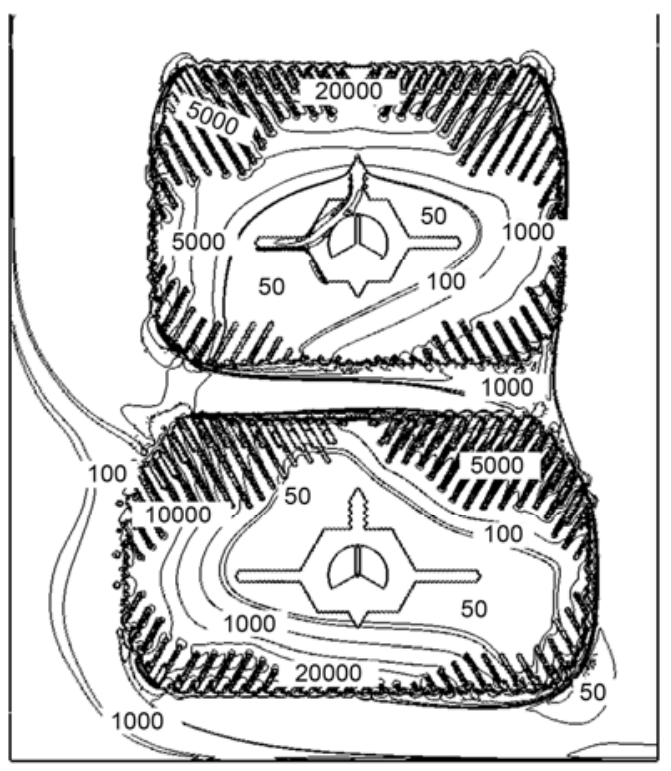

Figure 10 Mechanical energy losses of the condenser for tube arrangement $\mathrm{C}\left(\mathrm{W} / \mathrm{m}^{3}\right)$. 


\section{Conclusions}

(1) A theoretical analysis of the condenser shell side flow was used to develop a simplified expression for the mechanical energy loss rate. The mechanical energy loss consists of the mechanical energy dissipation due to the resistance of the tube bundle and that due to steam condensation. A well designed tube arrangement can reduce the mechanical energy loss and also the shell side pressure drop.

(2) Three typical tube arrangements were analyzed numerically. The results show that a higher condenser shell side pressure drop always corresponds to a larger total mechanical energy loss. The mechanical energy loss is concentrated in the periphery of the tube bundle, indicating that the flow pattern and the mechanical energy loss are markedly determined by the tube bundle profile. The condenser shell side pressure drop can be decreased by reducing the total mechanical energy loss by having the steam more uniformly entering the tube bundle so that the mechanical energy loss distribution in condenser is more uniform.

The work was supported by the National Natural Science Foundation of China (51036003) and the National Basic Research Program of China (2007CB206901).

1 Heat Exchange Institute. Standards for steam surface condensers. 2006

2 Zhang C, Sousa A, Venart J. Numerical simulation of different types of steam surface condensers. J Energ Resour-Asme, 1991, 113: 63-70

3 Zhang C, Sousa A, Venart J. The numerical and experimental study of a power plant condenser. J Heat Trans-T Asme, 1993, 115: 435-445

4 Zhang C. Numerical modeling using a quasi-3-dimensional procedure for large power plant condensers. J Heat Trans-T Asme, 1994, 116: 180-188

5 Malin M R. Modelling flow in an experimental marine condenser. Int Commun Heat Mass, 1997, 24: 597-608

6 Zhang C, Bokil A. A quasi-three-dimensional approach to simulate the two-phase fluid flow and heat transfer in condensers. Int J Heat Mass Tran, 1997, 40: 3537-3546

7 Sato K, Taniguchi A, Kamada T, et al. New tube arrangement of condenser for power stations. Fluids Therm Eng, 1998, 41: 752-758

8 Roy R P, Ratisher M, Gokhale V K. A computational model of a power plant steam condenser. J Energ Resour-Asme, 2001, 123: 81-91

9 Ramon I S, Gonzalez M P. Numerical study of the performance of a church window tube bundle condenser. Int J Therm Sci, 2001, 40: 195-204

10 Prieto M M, Suarez I M, Montanes E. Analysis of the thermal performance of a church window steam condenser for different operational conditions using three models. Appl Therm Eng, 2003, 23: 163-178

11 Guo Z Y, Zhu H Y, Liang X G. Entransy-A physical quantity describing heat transfer ability. Int J Heat Mass Tran, 2007, 50: 2545-2556

12 Wei S H, Chen L G, Sun F R. "Volume-point" heat conduction constructal optimization with entransy dissipation minimization objective based on rectangular element. Sci China Ser E, 2008, 51: 1283-1295

13 Wei S H, Chen L G, Sun F R. Constructal entransy dissipation minimisation for "volume-point" heat conduction without the premise of optimised last-order construct. Int J Exergy, 2010, 7: 627-639

14 Wei S H, Chen L G, Sun F R. Constructal entransy dissipation mini- mization for "volume-point" heat conduction based on triangular element. Therm Sci, 2010, 14: 1075-1088

15 Xiao Q H, Chen L G, Sun F R. Constructal entransy dissipation rate minimization for "disc-to-point" heat conduction. Chin Sci Bull, 2011, 56: 102-112

16 Xiao Q H, Chen L G, Sun F R. Constructal entransy dissipation rate minimization for umbrella-shaped assembly of cylindrical fins. Sci China Tech Sci, 2011, 54: 211-219

17 Xie Z H, Chen L G, Sun F R. Comparative study on constructal optimizations of $\mathrm{t}$-shaped fin based on entransy dissipation rate minimization and maximum thermal resistance minimization. Sci China Tech Sci, 2011, 54: 1249-1258

18 Xiao Q H, Chen L G, Sun F R. Constructal entransy dissipation rate minimization for heat conduction based on a tapered element. Chin Sci Bull, 2011, 56: 2400-2410

19 Chen L G, Wei S H, Sun F R. Constructal entransy dissipation rate minimization of a disc. Int J Heat Mass Tran, 2011, 54: 210-216

20 Meng J A, Liang X G, Li Z X. Field synergy optimization and enhanced heat transfer by multi-longitudinal vortexes flow in tube. Int $\mathrm{J}$ Heat Mass Tran, 2005, 48: 3331-3337

21 Chen Q, Ren J, Meng J A. Field synergy equation for turbulent heat transfer and its application. Int J Heat Mass Tran, 2007, 50: 5334-5339

22 Chen Q, Ren J X. Generalized thermal resistance for convective heat transfer and its relation to entransy dissipation. Chin Sci Bull, 2008, 53: 3753-3761

23 Chen Q, Wang M R, Pan N, et al. Optimization principles for convective heat transfer. Energy, 2009, 34: 1199-1206

$24 \mathrm{Wu} \mathrm{J}$, Liang X G. Application of entransy dissipation extremum principle in radiative heat transfer optimization. Sci China Ser E-Tech Sci, 2008, 51: 1306-1314

25 Guo J F, Cheng L, Xu M T. Entransy dissipation number and its application to heat exchanger performance evaluation. Chin Sci Bull, 2009, 54: 2708-2713

26 Liu X B, Meng J, Guo Z Y. Entropy generation extremum and entransy dissipation extremum for heat exchanger optimization. Chin Sci Bull, 2009, 54: 943-947

27 Xia S J, Chen L G, Sun F R. Optimization for entransy dissipation minimization in heat exchanger. Chin Sci Bull, 2009, 54: 3587-3595

28 Qian X D, Li Z X. Analysis of entransy dissipation in heat exchangers. Int J Therm Sci, 2011, 50: 608-614

29 Qian X D, Li Z, Li Z X. Entransy-dissipation-based thermal resistance analysis of heat exchanger networks. Chin Sci Bull, 2011, 56: 3289-3295

30 Guo J F, Xu M T, Cheng L. The influence of viscous heating on the entransy in two-fluid heat exchangers. Sci China Tech Sci, 2011, 54: $1267-1274$

31 Guo J F, Xu M T, Cheng L. Principle of equipartition of entransy dissipation for heat exchanger design. Sci China Tech Sci, 2010, 53: 1309-1314

32 Li X F, Guo J F, Xu M T, et al. Entransy dissipation minimization for optimization of heat exchanger design. Chin Sci Bull, 2011, 56: 2174-2178

33 Chen Q, Wu J, Wang M R, et al. A comparison of optimization theories for energy conservation in heat exchanger groups. Chin Sci Bull, 2011, 56: 449-454

34 Chen Q, Ren J X, Guo Z Y. Field synergy analysis and optimization of decontamination ventilation designs. Int J Heat Mass Tran, 2008, 51: 873-881

35 Chen Q, Meng J A. Field synergy analysis and optimization of the convective mass transfer in photocatalytic oxidation reactors. Int $\mathbf{J}$ Heat Mass Tran, 2008, 51: 2863-2870

36 Chen Q, Ren J X, Guo Z Y. Fluid flow field synergy principle and its application to drag reduction. Chin Sci Bull, 2008, 53: 1768-1772

37 Yakhot V, Orszag S A. Renormalization-group analysis of turbulence. Phys Rev Lett, 1986, 57: 1722-1724

Open Access This article is distributed under the terms of the Creative Commons Attribution License which permits any use, distribution, and reproduction in any medium, provided the original author(s) and source are credited. 